University of South Carolina

Scholar Commons

$12-2009$

\title{
Empirical Mode Decomposition Operator for Dewowing GPR Data
}

Bradley M. Battista

Adrian Addison

University of South Carolina - Columbia

Camelia C. Knapp

University of South Carolina - Columbia, camelia@geol.sc.edu

Follow this and additional works at: https://scholarcommons.sc.edu/geol_facpub

Part of the Earth Sciences Commons

\section{Publication Info}

Published in Journal of Environmental and Engineering Geophysics, Volume 14, Issue 4, 2009, pages 163-169.

Battista, B. M., Addison, A. D., \& C. C. Knapp. (2009). Empirical mode decomposition operator for dewowing GPR data. Journal of Environmental and Engineering Geophysics, 14 (4), 163-169.

(C)Journal of Environmental and Engineering Geophysics 2009, Society of Exploration Geophysicists

This Article is brought to you by the Earth, Ocean and Environment, School of the at Scholar Commons. It has been accepted for inclusion in Faculty Publications by an authorized administrator of Scholar Commons. For more information, please contact digres@mailbox.sc.edu. 


\title{
Empirical Mode Decomposition Operator for Dewowing GPR Data
}

\author{
Bradley M. Battista, Adrian D. Addison and Camelia C. Knapp \\ Department of Geological Sciences, University of South Carolina, 701 Sumter St. EWS 617, \\ Columbia, South Carolina 29208
}

\begin{abstract}
Signal processing tools available to ground penetrating radar data used for shallow subsurface imaging and hydrogeophysical parameter estimation are significantly handled using the same tools available to seismic reflection data. Overall, the same tools produce interpretable images from both data types, but particular noise (wow noise) in electromagnetic data must be removed before stable and accurate quantitative results can be produced. Wow noise is an inherent, nonlinear electromagnetic interference and a significant component of GPR data. Further, the nonlinear and non-stationary nature of wow noise provides a strong argument for preprocessing radar traces with time-domain operators. Time-domain operators designed for nonlinear signals are under increasing development for both electromagnetic and acoustic signal processing. This work demonstrates optimal wow noise removal from ground penetrating radar data using the empirical mode decomposition. The technique provides a data-driven approach to empirically dewowing GPR data.
\end{abstract}

\section{Introduction}

\section{Premise}

Ground penetrating radar (GPR) has a strong foundation in environmental engineering and groundwater investigation, but it also has gained widespread use for shallow sedimentary and stratigraphic studies. Like its acoustic analogue, electromagnetic signal processing governs data application. Common geophysical applications range from basic subsurface imaging to high-order geostatistical evaluation. The former depends more so on how the data are acquired while the latter is significantly dependent on how data are processed.

Both electromagnetic and acoustic signal processing follow similar conditions. The fundamental condition is that oscillatory source pulses are modulated by the changing medium through which they propagate. Dix (1949) describes such oscillatory pulses, or "seismic pulses," as having no amplitude at their beginning or end and exhibiting a zero local mean throughout. Seismic pulses, however, naturally exist in the presence of noise. Shannon (1949) addresses noise as it applies to communication theory, the governing theory over digital signal processing. Slepian (1976) expands this to include "effective" bandwidth, a system's dynamic range. To summarize decades of theory into a fundamental concept, time-limited signals are band-unlimited in frequency and they occur simultaneously with potentially unknown noise sources. The apparent time- frequency paradox stems from the Heisenberg's Uncertainty Principle and requires a signal of limited duration possess infinite bandwidth. This means that no timelimited signal can be digitally recorded and processed without some degradation of signal quality; this occurs near and beyond the Nyquist frequency (Shannon, 1948). This is the most fundamental limitation of digital signal processing, and it is often too subtle to inhibit most geophysical applications. However, increasing technology and advanced application give more weight to these basic limitations. For example, high-resolution marine seismic profiling (McGee, 2000) has been coupled with empirical, nonlinear signal processing (Battista et al., 2007) in response to a problem noted by Hardage and Roberts (2006) that seismic technology must advance before seismoacoustic identification of buried gas hydrates is possible. McGee et al. (2008) and following works undergoing review may have overcome this obstacle. Similarly, GPR data processing stands to gain from the same advancements in digital signal processing.

The Need for Advancement

Advancements in GPR data processing following geotechnical studies such as Davis and Annan (1989) and Oldenborger et al. (2004) allow for quantitative analysis and estimation of subsurface composition. However, signal attribute modulation in response to anisotropic dielectric permittivity demands careful 
Journal of Environmental and Engineering Geophysics

consideration of even the most basic processing utilities (Kutrubes et al., 1994). It is of utmost importance to preserve phase, frequency, and amplitude before using any signal attribute to estimate subsurface composition. Gerlitz et al. (1993) noted that non-stationary wow noise inherent to GPR traces warrants the use of advanced time-domain utilities to appropriately identify and remove it. More importantly, they observed the need for a data-driven approach to optimally filter wow noise and ensued their investigation with an average length residual median filter. In hindsight, it is apparent that the method of dewowing GPR data governs preservation of signal attributes, and significant advancements in data-driven signal processing may better preserve these signal attributes and allow for improved determination of dielectric constants.

This work demonstrates an alternative timedomain operator, the empirical mode decomposition (EMD), for dewowing GPR data such that traditional seismic reflection processing tools may follow, and meaningful quantitative results may be produced (Addison et al., 2009). The EMD, introduced by Huang et al. (1998), is a data-driven process that is well suited for handling data from both stochastic and astochastic processes. Therefore, our objective is to demonstrate that it can optimally dewow GPR data without the need for manually optimizing a filter for each trace.

\section{Data and Processing}

GPR data were collected with a Sensors \& Software PulseEkko 100 system. Acquisition employed a $100 \mathrm{MHz}$ antennae with a sample interval of 800 picoseconds $(1,250 \mathrm{MHz})$. Data were acquired at the Marine Corps Air Station in Beaufort, South Carolina. The geologic setting is quite simple, horizontal layers of Pleistocene sands overlying Eocene Ocala limestone. The sands are subdivided into three layerssurface, upper, and basal sand units. The water table commonly lies $2-3 \mathrm{~m}$ below a nearly flat ground surface. The site allows for high-quality data acquisition in an area having pre-existing, complimentary data available. Among these data are velocity derived from a borehole located on the GPR line approximately $20 \mathrm{~m}$ from its beginning. The borehole velocity was derived from vertical radar data which is extrapolated along the horizons of the GPR data. In this manner, the GPR data set serves as a nice means for studying dewowing techniques and for estimating dielectric constants as the geologic and hydrogeologic stratigraphy are simple and well known. These efforts are carried out following EMD dewow by Addison et al. (2009).

A minimal processing flow for GPR traces involves bandwidth modulation and amplitude recov- ery. Bandwidth is adjusted to remove noise while amplitude recovery restores energy lost to attenuation during signal transmission. The most significant role of bandwidth modulation is to reduce wow noise. Optionally, deconvolution may be added to the processing flow, but Fisher et al. (1992) and Kutrubes et al. (1994) noted that deconvolution can be subjective and may not be necessary for high quality GPR traces. The high sample rate and data quality of GPR traces generally provide a compact wavelet that is minimally improved if not degraded by deconvolution, and it is not performed here. Any following processing would depend on the method of GPR acquisition. This work addresses only the dewowing stages of bandwidth modulation because it is essential to any GPR data.

Dewowing with Empirical Mode Decomposition

The EMD used here is a modified version, adapted for use with seismic reflection signals by Battista et al. (2007). The main adaptations are to statistical rules and curve-fitting precision such that the EMD is biased toward the seismic pulses described by Dix (1949). A significant application of the EMD in that work, and the direct application here, is as a time-domain detrending tool. To summarize, the EMD separates a signal into several subsignals of varying and possibly overlapping frequency content. The first sub-signal produced contains the highest frequencies, while the last contains the lowest frequencies, as shown in Fig. 1. The summation of these sub-signals, also known as intrinsic mode functions (IMF), reproduces the original signal (Fig. 2). IMFs must possess two distinct characteristics: the total number of extrema (peaks and troughs) in an IMF must not differ from the total number of zero crossings by more than one, and the IMF must not contain a nonzero mean. The method of producing IMFs is known as "sifting" (Huang et al., 1998).

Sifting is an iterative detrending process through which all low-frequency trends preventing a [sub]signal from fitting the criteria for an IMF are removed from a given mode (Huang et al., 1998). Sifting works by first separating a signal's extrema into peaks and troughs. A cubic-spline curve fit is created for each set of extrema, and the signal is effectively enveloped within the two curve fits. The average of the two curve fits is taken as a function of time, and is referred to as the "mean" spline (Fig. 3). The mean spline is subtracted from the subsignal to produce a new signal, and the process is repeated until the criteria for an IMF are met. In this manner, a signal that already fits the criteria for an IMF will resist sifting and produce no further output. In contrast, real-world signals rarely exhibit a mean spline of exactly zero, and a final stopping criterion must be set to determine when sifting has effectively produced an IMF. This criterion is a predetermined root mean square 
Battista et al.: Empirical Mode Decomposition Operator for Dewowing GPR Data

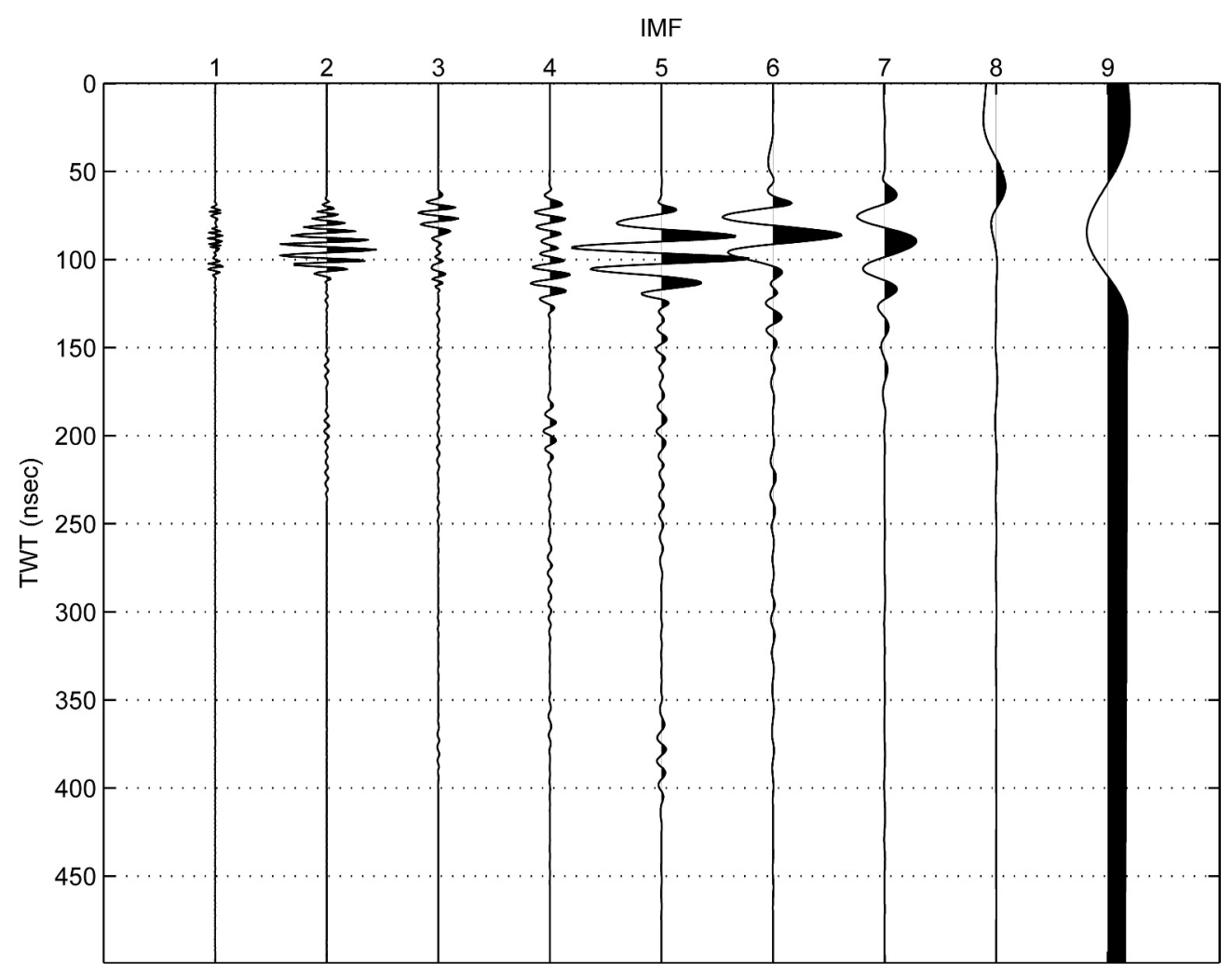

Figure 1. Intrinsic mode functions (IMF) produced by the empirical mode decomposition. Wow noise is apparent in IMFs 8 and 9.

(RMS) tolerance between two consecutive components of sifting. Let $s_{0}(t)$ be sifted once to yield its first component, $s_{1}(t)$, by removing its mean spline $m_{0}(t)$. The RMS values are determined for $s_{0}(t)$ and $s_{1}(t)$, and the RMS difference is compared to the predetermined tolerance. Sifting continues according to Eq. (1) if the difference between RMS values is greater than the tolerance:

$$
\begin{aligned}
& R M S_{(n-1)}-R M S_{(n)}>\text { tolerance, } \\
& s_{n+1}(t)=s_{n}(t)-m_{n}(t),
\end{aligned}
$$

where $n$ is the sifting iteration. The final sifted result for a given IMF contains only the highest frequency components of the input signal that do not cause it to violate the IMF criteria. Subtracting the first IMF from the original signal produces a new input signal which may be sifted to form a second IMF. Sifting and production of IMFs continues until the process is manually terminated or the remaining residual contains at most three extrema. Therefore, IMFs relate to the original signal in accordance with Eq. (2):

$$
c_{0}(t)=\left(\sum_{n=1}^{N} c_{n}(t)\right)+r(t),
$$

where $c_{0}(t)$ is the original signal, $c_{n}(t)$ are IMFs, $r(t)$ is a residual, if any, and $n$ and $N$ are the IMF number and total number of IMFs, respectively.
The strength of EMD as a detrending operator is only partially determined by the statistical controls used during sifting. Battista et al. (2007) configured rootmean square stopping criteria and spline controls for optimal decomposition of seismic reflection traces. Added strength for EMD as a detrending operator, however, lies in how the IMFs are handled before reconstructing a trace using Eq. (2). Rilling et al. (2004), $\mathrm{Wu}$ and Huang (2004), and Battista (2008) expand the detrending capabilities of EMD by either exclusion of IMFs or amplitude modulation of IMFs. Exclusion of IMFs is not recommended unless a very high quality EMD is performed because it may introduce a phase shift. Instead, amplitude modulation of IMFs affords the ability to suppress time-varying components of a signal without significantly degrading the remaining components. This also allows for further adaptation and automation of the EMD. In short, a user can configure the EMD and post-EMD modulate the IMFs based on a predetermined time-varying condition such as wow noise. Wow noise in this study, for example, nearly always decomposes into the last two IMFs, and these can be scaled to lower amplitude before trace reconstruction. However, the results shown in the following section determined wow noise by checking which IMFs contained $30 \mathrm{MHz}$ content and scaling them to near- 
Journal of Environmental and Engineering Geophysics

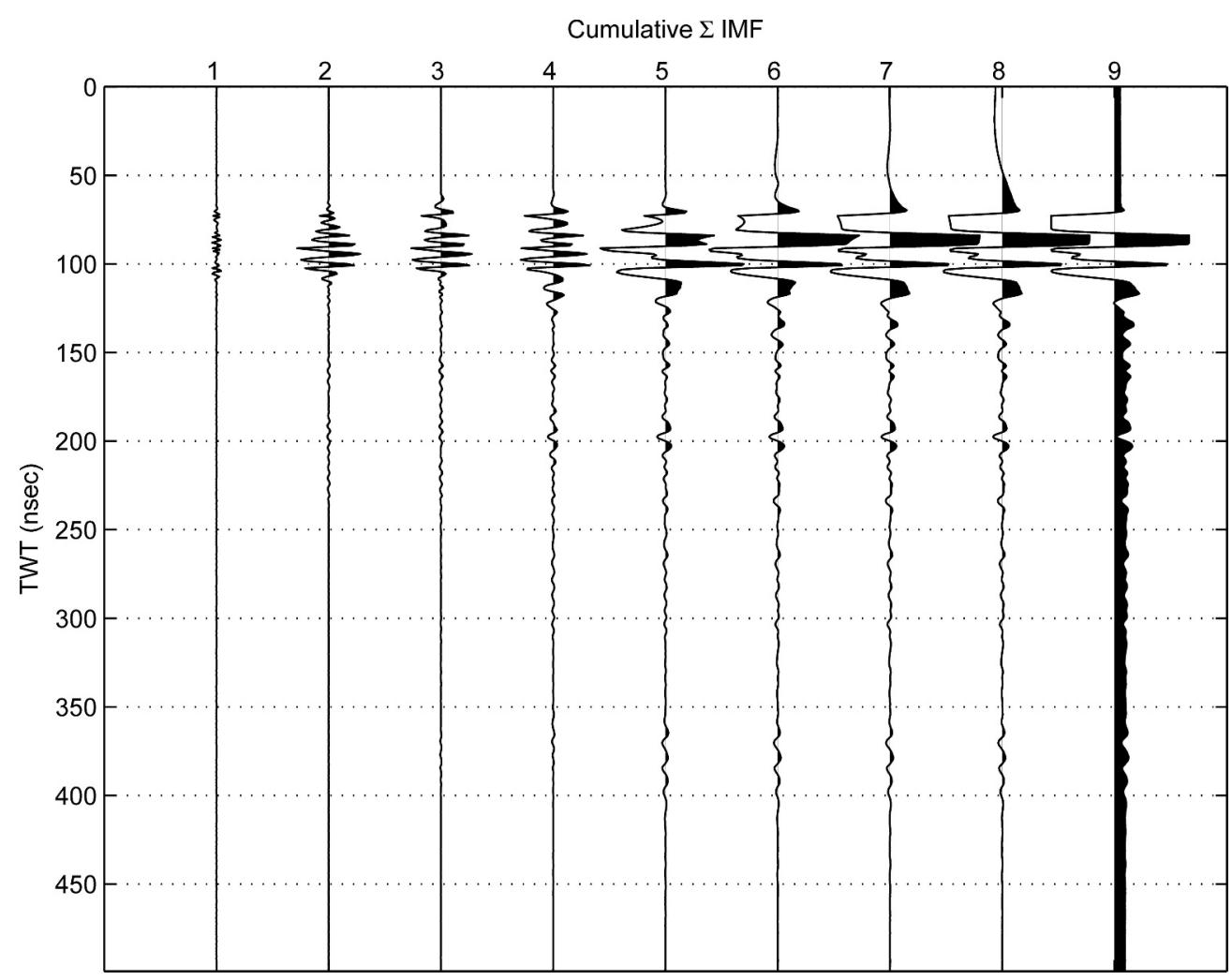

Figure 2. Intrinsic mode functions (IMF) produced by the empirical mode decomposition. The sum of all modes will produce the original input GPR trace, as seen in the right-most trace.

zero amplitude. The following section compares this empirical, data-driven approach to the residual median filter described by Gerlitz et al. (1993).

\section{Dewow Comparison}

A residual median filter (RMF) works like a moving average except it takes the median instead of the average for a given window length of data. It is preferred to a moving average because it is more respecting of edge effects. Gerlitz et al. (1993) compare moving and median filters to a bandpass filter for dewowing GPR traces. The RMF performs the best for their study, but for true dewow it requires that each trace be manually analyzed to determine the appropriate window size. This is a significant disadvantage considering the characteristics of wow noise may vary from trace to trace. This is

\section{Upper Spline}

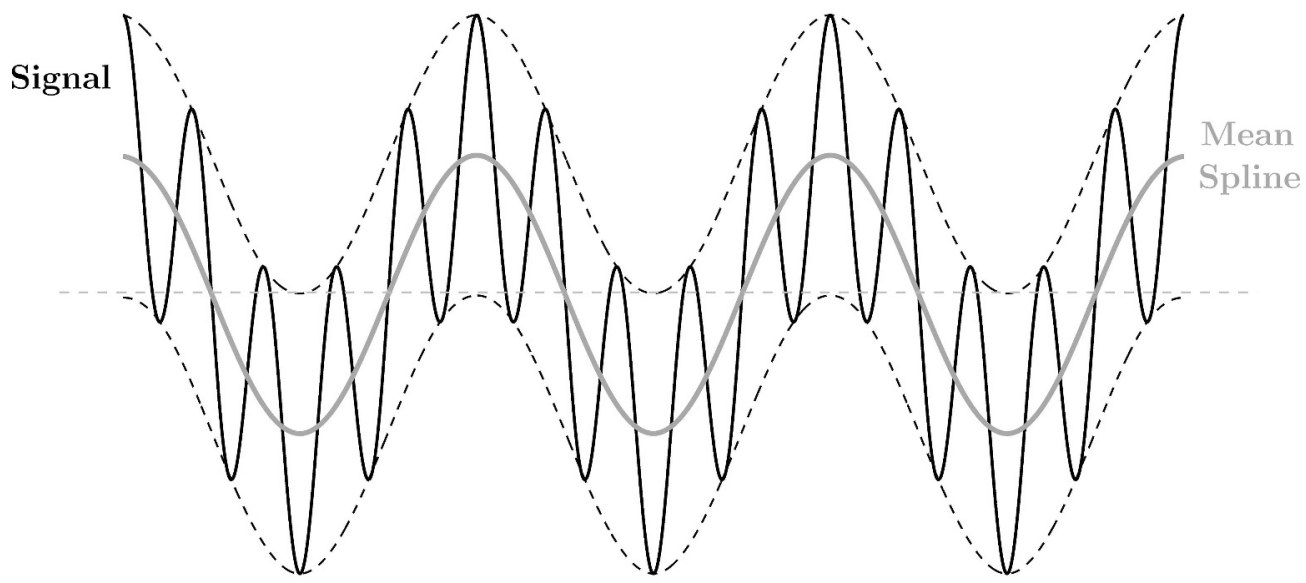

Lower Spline

Figure 3. Mean spline produced as the mean of the upper and lower cubic spline fits to a time series. 


\section{Battista et al.: Empirical Mode Decomposition Operator for Dewowing GPR Data}

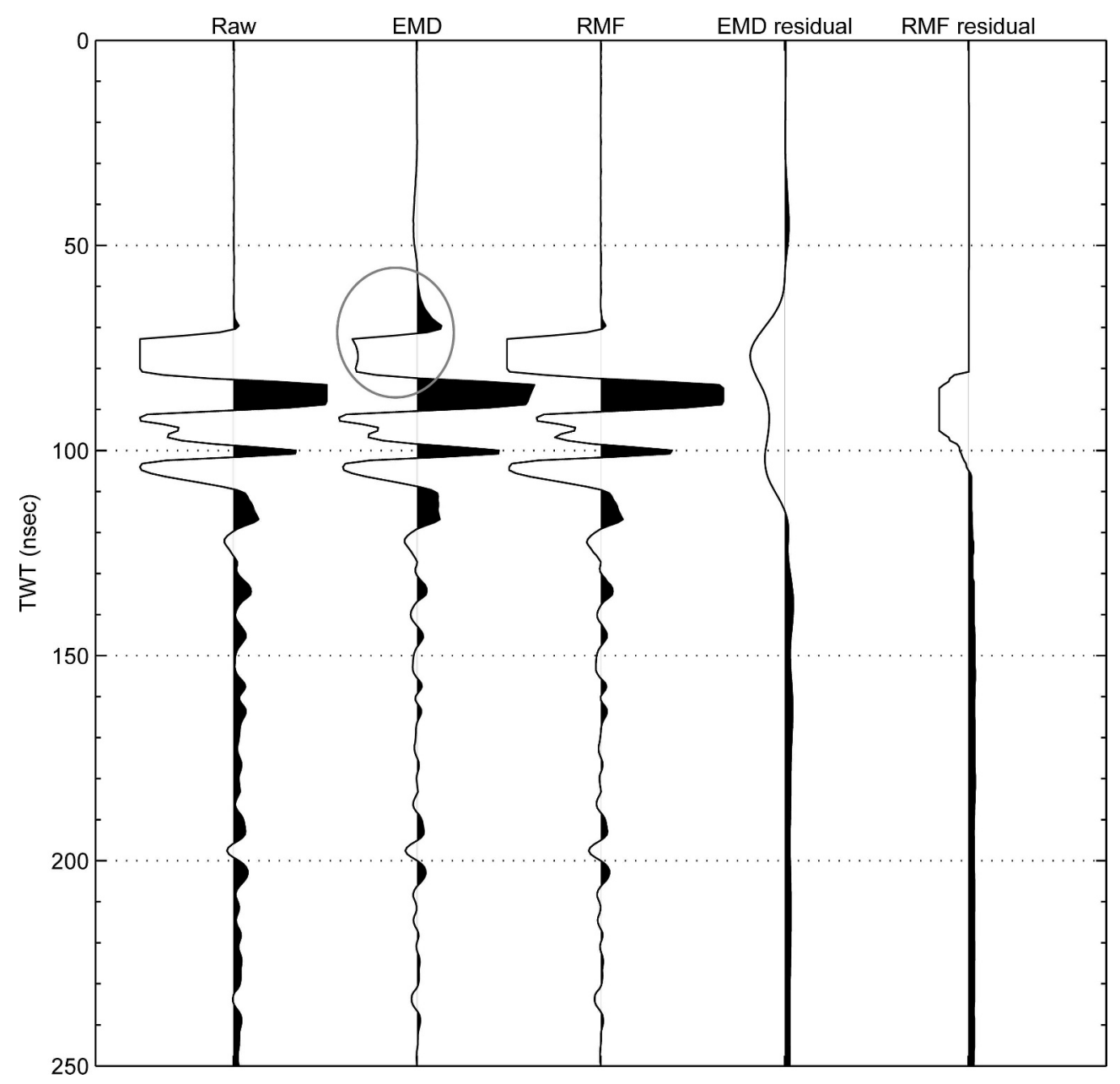

Figure 4. Dewow comparison of GPR trace. The EMD and RMF results are nearly identical, but the EMD filter was entirely data driven while the RMF was chosen. The encircled area of the EMD-produced trace shows the EMD's sensitivity to clipping.

the primary advantage for using EMD in place of RMF. EMD adapts from trace to trace and carries no criterion for window size. The time-varying characteristics of wow noise are different from the geologic information, and that alone allows the EMD to separate it. Figure 4 provides visual comparison of EMD and RMF dewow. The results are nearly identical where data are not clipped. The curve-fitting nature of EMD introduces error wherever amplitude saturates and a signal is clipped. This is not the downfall of the technique, however, since clipping is a loss of data that should be avoided in practice. Although, EMD is able to handle very weak data, often as weak as $60 \mathrm{~dB}$ down (Battista, 2008), which allows for acquisition to avoid settings requiring such sacrifices of shallow information as data clipping in order to obtain strong signals further from the source. Nonetheless, results of the data-driven EMD closely resemble those of RMF without the need for trace-by-trace validation. In comparison, the clipping response of the RMF is also affected. The residuals from both EMD and RMF show that wow noise for the clipped area is different. The remainder of each residual is the same, which suggests the two filters are very similar even though one was data-derived (EMD) while the other was user supplied (RMF).

Demonstrating the technique for an entire GPR line further validates the above technique and comparison. Figure 5 shows raw GPR traces for the entire line. There is little stratigraphic information present as the strong bias toward positive amplitude from wow noise masks subsurface information. The application of a RMF dewow shown in Fig. 6 shows significant reduction of the wow noise. Figure 7 demonstrates a similar result, but using EMD for dewowing. Comparison of the two figures provides a similar result as in Fig. 4. However, to reiterate the point, the EMD dewow is data-driven while the RMF requires trace-by-trace optimization. The EMD dewow could follow a similar 


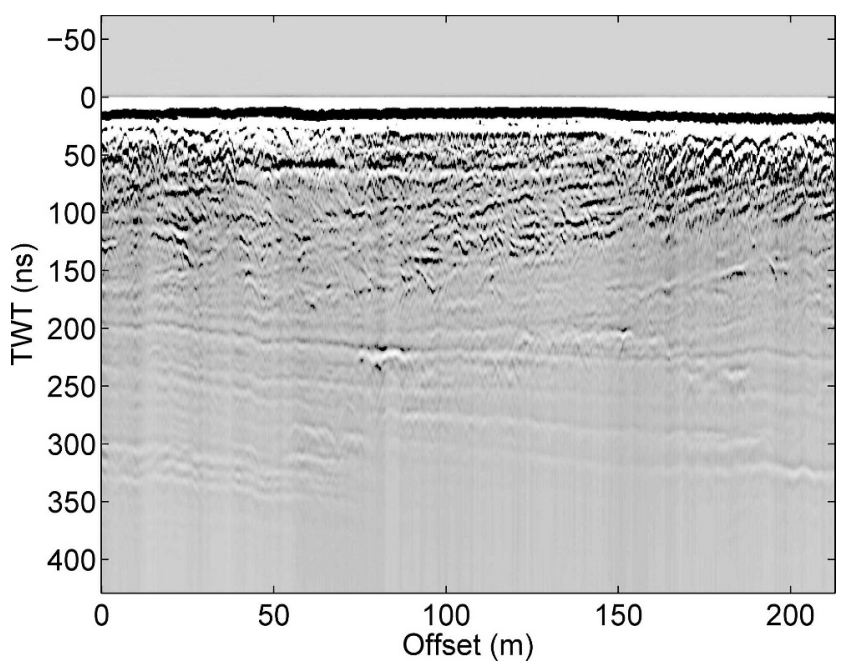

Figure 5. Raw GPR traces containing wow noise. Notice the bright white energy near $t_{0}$ and the strong bias toward the gray color in the deeper section. This is wow noise biasing the subsurface response of the source to positive values.

requirement, but we chose to suppress IMFs having $30 \mathrm{~Hz}$ content versus manual picking.

\section{Conclusion}

The overview of this work demonstrates that the EMD is a well-suited tool for processing GPR data. The leading approach to removing wow noise by residual median filtering (Gerlitz et al., 1993) is suitable, but demands trace-by-trace interaction to truly dewow GPR data. Substituting residual median filtering with EMD precludes this demand. It also affords the ability to track, isolate, and manipulate any time-varying characteristic of seismic pulses while preserving the attributes

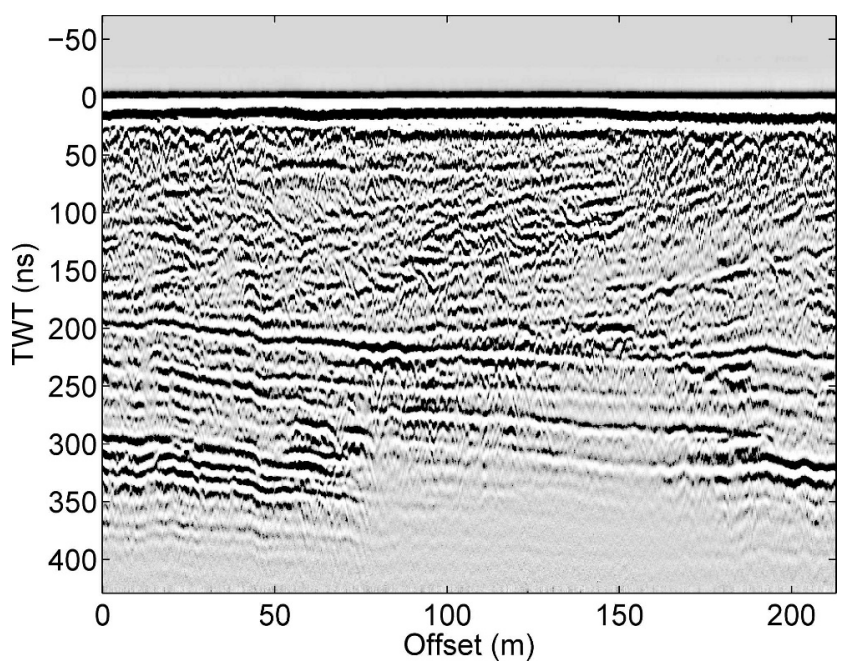

Figure 6. GPR traces dewowed using a residual median filter.

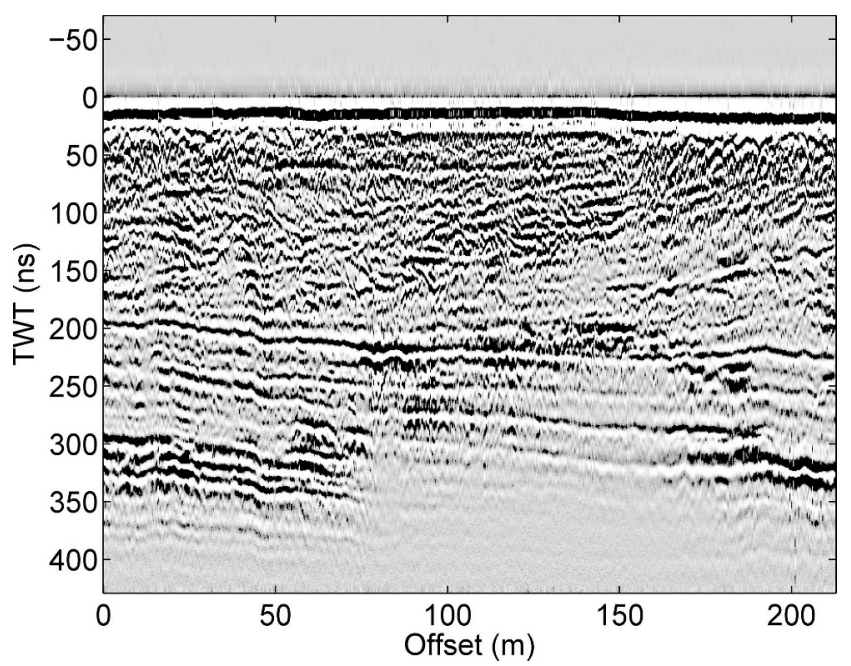

Figure 7. GPR traces dewowed using an empirical mode decomposition filter.

of the remaining signal (Battista, 2008; Addison et. al., 2009). The implications of such a utility are not fully understood, but minimal usage as a detrending utility is more than appropriate for adequately dewowing GPR data.

\section{Acknowledgments}

This work is made possible by support and cooperation from the Dept. of Interior (Minerals Management Service), Dept. of Energy (National Energy Technology Laboratory), Dept. of Commerce (National Oceanic and Atmospheric Administration), State of South Carolina (Marine Air Corps Station at Beaufort), University of Mississippi (Center for Marine Resources and Environmental Technology), and University of South Carolina (Geophysical Exploration Laboratory, Tectonics and Geophysics Laboratory, and Earth Sciences and Resources Institute).

\section{References}

Addison, A., Battista, B.M., and Knapp, C., 2009, Improved hydrogeophysical parameter estimation from empirical mode decomposition processed ground-penetrating radar data: Journal of Environmental and Engineering Geophysics, 14(4) 00-00.

Battista, B.M., 2008. Advanced nonlinear signal processing tools for use with high-resolution seismic reflection and ground-penetrating radar data: $\mathrm{PhD}$ thesis, University of South Carolina, Columbia, South Carolina, 98 pp.

Battista, B.M., Knapp, C., McGee, T.M., and Goebel, V., 2007, Application of the empirical mode decomposition and Hilbert-Huang transform to seismic reflection data: Geophysics, 72(2) 29-37.

Davis, J., and Annan, A.P., 1989, Ground-penetrating radar for high resolution mapping of soil and rock stratigraphy: Geophysical Prospecting, 37, 531-551. 


\section{Battista et al.: Empirical Mode Decomposition Operator for Dewowing GPR Data}

Dix, H., 1949, On the minimum oscillatory character of spherical seismic pulses: Geophysics, 14, 17-20.

Fisher, E., McMechan, G.A., and Annan, A.P., 1992, Acquisition and processing of wide-aperture ground-penetrating radar data: Geophysics, 57, 495-504.

Gerlitz, K., Knoll, M.D., Cross, G.M., Luzitano, R.D., and Knight, R., 1993, Processing ground penetrating radar data to improve resolution of near-surface targets: in Proceedings: Symposium on the Application of Geophysics to Environmental and Engineering Problems, 561-574.

Hardage, B.A., and Roberts, H.H., 2006, Gas hydrate in the Gulf of Mexico: What and where is the seismic target?: The Leading Edge, 25, 566-571.

Huang, N., Shen, Z., Long, S., Wu, M., Shih, E., Zheng, Q., Tung, C., and Liu, H., 1998, The empirical mode decomposition method and the Hilbert spectrum for non-stationary time series analysis: Proceedings of the Royal Society of London, A454, 903-995.

Kutrubes, D.L., Zhang, J., and Mackie, R.L., 1994, Determination of GPR processing capabilities using ProMAX: in Expanded Abstracts: Proceedings of the Institute of Electrical and Electronics Engineers Dual-Use Technologies and Applications Conference, 439-446.

McGee, T., 2000, Pushing the limits of high-resolution in marine seismic profiling: Journal of Environmental and Engineering Geophysics, 5, 43-53.
McGee, T., Woolsey, J., Lapham, L., Kleinberg, R., Macelloni, L., Battista, B., Knapp, C., Caruso, S., Goebel, V., Chapman, R., and Gerstoft, P., 2008, Structure of a carbonate/hydrate mound in the northern Gulf of Mexico Presented at the 6th International Conference on Gas Hydrates.

Oldenborger, G.A., Knoll, M.D., and Barrash, W., 2004, Effects of signal processing and antenna frequency on the geostatistical structure of ground-penetrating radar data: Journal of Environmental and Engineering Geophysics, 9, 201-212.

Rilling, G., Flandrin, P., and Gonçalvès, P., 2004, Detrending and denoising with empirical mode decomposition Presented at the Eusipco, 12th European Signal Processing Conference.

Shannon, C., 1948, A mathematical theory of communication: The Bell System Technical Journal, 27, 379-423, 623-656.

Shannon, C., 1949, Communication in the presence of noise: Proceedings of the Institute of Radio Engineers, 10-21.

Slepian, D., 1976, On bandwidth: Proceedings of the Institute of Electrical and Electronics Engineers, 292-300.

Wu, Z., and Huang, N., 2004, A study of the characteristics of white noise using the empirical mode decomposition method: Proceedings of the Royal Society of London, 460, 1597-1611. 